\title{
Role of Kruppel-like factor 4 in regulating inhibitor of apoptosis-stimulating protein of p53 in the progression of gastric cancer
}

\author{
LULU WANG $^{1}$, YAN LI ${ }^{1}$, LUCHUN LI ${ }^{1}$, ZHIJUAN WU ${ }^{1}$, YONGZHONG WU ${ }^{2}$, HUIWEN MA ${ }^{1}$, \\ HUIQING YU ${ }^{1}$, DAN YANG ${ }^{1}$ and DONGLIN WANG ${ }^{1}$ \\ Departments of ${ }^{1}$ Oncology and ${ }^{2}$ Radiotherapy, Chongqing Cancer Institute, Chongqing 400030, P.R. China
}

Received June 26, 2017; Accepted December 8, 2017

DOI: $10.3892 / \mathrm{ol} .2018 .8203$

\begin{abstract}
Gastric cancer (GC) remains one of the leading causes of cancer-associated mortality. The overexpression of inhibitor of apoptosis-stimulating protein of p53 (iASPP) has been detected in GC tissues but the function of iASPP in the viability of GC cells and its underlying molecular mechanism remains unknown. Kruppel-like factor 4 (KLF4) is a tumor suppressor gene in GC and it may interact with p53. iASPP is an evolutionarily conserved inhibitor of p53, whereas KLF4 may be negatively associated with iASPP in GC. However, whether KLF4 has regulatory effects on iASPP remains to be investigated. The objective of the present study was to examine the function of iASPP and KLF4 in the proliferation of GC cells and to determine whether KLF4 has regulatory effects on iASPP. It was demonstrated that iASPP was upregulated and KLF4 was downregulated in GC cell lines. Downregulation of iASPP inhibited the proliferation and colony formation ability, and promoted the apoptosis of GC cells. Additionally, upregulation of KLF4 inhibited the proliferation and colony formation ability, and promoted apoptosis of GC cells. Furthermore, upregulation of KLF4 inhibited the expression of iASPP. Upregulation of iASPP following overexpression of KLF4 reversed the KLF4-mediated effects in GC cells. In vivo upregulation of KLF4 or downregulation of iASPP inhibited the growth of tumors, whereas upregulation of iASPP promoted the growth of tumors. In conclusion, iASPP may act as an oncogene that promotes the proliferation of GC cells. The results demonstrated that KLF4 was a negative regulatory factor of iASPP and that overexpression of iASPP inhibited the effects of KLF4. Thus, downregulation of KLF4
\end{abstract}

Correspondence to: Professor Donglin Wang, Department of Oncology, Chongqing Cancer Institute, 181 Han Yu Road, Chongqing 400030, P.R. China

E-mail: wanglulu198819@163.com

Key words: gastric cancer, inhibitor of apoptosis-stimulating protein of p53, Kruppel-like factor 4, p53 in GC may lead to overexpression of iASPP and promote the development of cancer.

\section{Introduction}

Gastric cancer (GC) represents a major public health threat globally. GC develops due to various molecular abnormalities, including the activation of a number of oncogenes, the inactivation of several cancer suppressor genes and the abnormal regulation of various growth factors and their receptors $(1,2)$.

Inhibitor of apoptosis-stimulating protein of p53 (iASPP) is a member of the ASPP family that acts as a negative regulator of the wild-type p53 $(3,4)$. Wild-type p53 is a well-known tumor suppressor that regulates the cell cycle and apoptosis $(5,6)$. iASPP directly binds to the DNA-binding domains of p53 and inhibits its function, leading to abnormal cell proliferation and malignant transformation (7). iASPP may serve as a pro-oncogene since abnormal overexpression of iASPP has been detected in several types of human cancer, including breast carcinoma (8), acute leukaemia (9), lung cancer (10) and hepatocellular carcinoma (11). Previous studies demonstrated that the expression of iASPP was significantly increased in GC tissues compared with that in the corresponding normal tissues (12). Additionally, wild-type p53 was detected in $2 / 3$ of GC cases $(12,13)$. This evidence indicated that iASPP may act as an oncogene in GC. It may be possible to inhibit wild-type p53 to promote the development of GC. To the best of our knowledge, few studies have investigated the function of iASPP in human GC cells (12). Previous studies have focused on the downstream activities of iASPP targets $(14,15)$. Therefore, the upstream molecular mechanism of iASPP remains unclear.

Kruppel-like factor 4 (KLF4) is a zinc-finger transcription factor that contains Cys2-His2 motifs and is highly expressed in the gastrointestinal tract (16). It has been demonstrated that KLF4 exhibits dual functions in carcinogenesis since it may act as a tumor suppressor gene and as an oncogene (17). KLF4 has been demonstrated to function as an oncogene in several types of tumor, including breast cancer, head and neck squamous cell cancer, and oral squamous cancer, since its expression was upregulated and thus promoted the development and progression of these types of cancer (17-19). However, KLF4 may also 
function as a tumor suppressor since downregulation of KLF4 has been detected in colorectal, gastric, bladder, oesophageal and cervical cancer (17,20-24). Previous studies demonstrated that knockdown of KLF4 in the stomach increased cell proliferation and triggered the formation of pre-cancerous lesions. Additionally, progressive downregulation of KLF4 was associated with advanced tumor stages and increased metastases in patients with GC. However, increased expression of KLF4 in patients with GC was associated with increased survival rate $(25)$.

KLF4 may regulate cellular proliferation, apoptosis and differentiation by binding to the CACCC or GC-rich DNA sequences $(26,27)$. Previous studies have indicated an interaction between KLF4 and p53. KLF4 may regulate the transcription of the $\mathrm{p} 53$ promoter, whereas p53 may regulate the expression of KLF4 $(28,29)$. The feedback loop between KLF4 and p53 may regulate cell apoptosis. Since iASPP is an evolutionarily conserved inhibitor of p53, the aim of the present study was to investigate whether there is a functional association between KLF4, iASPP and p53.

The present study examined whether the expression of the transcription factor KLF4 regulated iASPP in the gastric mucosa. Additionally, since KLF4 and iASPP interact with p53, it was hypothesized that KLF4 may exhibit an anticancer effect through regulating the expression of iASPP in GC. Additionally, the effects of downregulating the expression of KLF4 in cellular proliferation and differentiation and in regulating the expression of iASPP in GC were investigated.

The precise molecular mechanism underlying the effects of KLF4 and iASPP remains unclear and future studies are required to unravel the effects of this signaling pathway in GC. The present study examined the expression of KLF4 and iASPP in GC cells and investigated their underlying molecular mechanism in the proliferation and apoptosis of GC cells.

\section{Materials and methods}

Cell lines. The normal gastric mucosa GES1 cell line was obtained from the Type Culture Collection of the Chinese Academy of Sciences (Shanghai, China). GES1 cells were cultured in Dulbecco's modified Eagle's medium (DMEM; Thermo Fisher Scientific, Inc., Waltham, MA, USA) supplemented with $10 \%$ foetal bovine serum (FBS; Thermo Fisher Scientific, Inc.) without antibiotics at $37^{\circ} \mathrm{C}$ in a humidified atmosphere containing 5\% $\mathrm{CO}_{2}$. GC cell lines (MKN45, BGC823 and SGC7901) were obtained from the American Type Culture Collection (Manassas, VA, USA). MKN45 is a cell line expressing wild-type p53 (30), SGC7901 is a cell line expressing mutant-type p53 (31) and BGC823 is a cell line that can express either wild-type p53 or mutant-type p53 $(32,33)$. The GC cells were routinely cultured in RPMI-1640 (Thermo Fisher Scientific, Inc.) medium supplemented with $10 \%$ FBS (Thermo Fisher Scientific, Inc.) without antibiotics at $37^{\circ} \mathrm{C}$ in a humidified atmosphere containing $5 \% \mathrm{CO}_{2}$.

Lentiviral transfection. To upregulate the KLF4 and iASPP expression in MKN45 cells, KLF4-overexpression-shRNA, iASPP-overexpression-shRNA and negative control-shRNA lentiviral were transfected. To downregulate the iASPP expression in MKN45 cells, the iASPP-inhibition-shRNA and negative control-shRNA lentiviral were transfected with Lipofectamine 2000 (Invitrogen; Thermo Fisher Scientific Inc., Waltham, MA, USA) at a final concentration of 100-200 nM, according to the manufacturer's instructions. All lentiviral suspensions were purchased from Shanghai Genepharma Co., Ltd. (Shanghai, China). To perform the lentiviral transfection, the target GC cells were grown to 20-30\% confluence in a 6 -well plate $\left(\sim 5 \times 10^{4}\right.$ cells/well $)$ and incubated for $12 \mathrm{~h}$ prior to transfection. During the transfection, the medium was replaced with a supernatant fluid that contained an appropriate titre of the virus (multiplicity of infection=virus number/cell number=20) and supplemented with $5 \mu \mathrm{g} / \mathrm{ml}$ polybrene (Sigma-Aldrich; Merck KGaA). Following incubation for $12 \mathrm{~h}$ at $37^{\circ} \mathrm{C}$, the supernatant containing lentivirus particles was replaced with fresh media. At $48 \mathrm{~h}$ post-transfection, cells were selected using puromycin $(2 \mathrm{mg} / \mathrm{ml}$; Beijing Solarbio Science \& Technology Co., Ltd., Beijing, China) to establish the stable clone cell lines. A total of $72 \mathrm{~h}$ later, the transfection efficiency of cells was observed using fluorescence microscopy (Leica Microsystems GmbH, Wetzlar, Germany). Cells were harvested $96 \mathrm{~h}$ after transfection, and the KLF4 or iASPP expression was evaluated using western blot analysis. Once the detections above were performed, the cells were used for further analysis.

Reverse transcription-polymerase chain reaction (RT-PCR). Total RNA was extracted from GES1, MKN45, BGC823 and SGC7901 cells using TRIzol reagent (Takara Bio, Inc., Otsu, Japan), according to the manufacturer's protocol. cDNA was amplified using the PrimeScript First Strand cDNA Synthesis kit (Takara Bio, Inc.). qPCR was performed using the SYBRR Premix Ex Taq ${ }^{\mathrm{TM}}$ II system (Takara Bio, Inc.) and the Bio-Rad CFX96 ${ }^{\mathrm{TM}}$ Real-time system (Bio-Rad Laboratories, Inc., Hercules, CA, USA). The following primers were used: iASPP, 5'-GCGGTGAAGGAGATGAACGA-3' (forward) and 5'-GCG GTGAAGGAGATGAACGA-3' (reverse); KLF4, 5'-CCCGGA TCCATGGCTGTCAGCGACGCGCTG-3' (forward) and 5'-CCCGAATTCTTAAAATGCCTCTTCATGTGTAAG-3' (reverse); and $\beta$-actin, 5'-CCACGAAACTACCTTCAACTC C-3' (forward) and 5'-GTGATCTCCTTCTGCATCCTGT-3' (reverse). $\beta$-actin was used as an internal control. The thermocycling conditions were as follows: Initial denaturation of $94^{\circ} \mathrm{C}$ for $60 \mathrm{sec}$, followed by 40 cycles of $94^{\circ} \mathrm{C}$ for $40 \mathrm{sec}$ and $60^{\circ} \mathrm{C}$ for $40 \mathrm{sec}$, and final extension of $72^{\circ} \mathrm{C}$ for $6 \mathrm{~min}$. Relative expression levels of the genes were determined using $2^{-\Delta \Delta \mathrm{Cq}}$ analysis (34).

Western blot analysis. MKN45 cells were washed with ice-cold PBS and lysed using a radioimmunoprecipitation assay buffer (Beyotime Institute of Biotechnology, Shanghai, China) for $30 \mathrm{~min}$ on dry ice. The lysates were collected with a rubber scraper, sonicated and centrifuged at 12,000 x g ( $4^{\circ} \mathrm{C}$ for $20 \mathrm{~min}$ ). The concentrations of the total proteins were determined using a bicinchoninic acid assay (Thermo Fisher Scientific, Inc.). Each sample was adjusted to $2 \mu \mathrm{g} / \mu \mathrm{l}$ and a $20 \mu$ volume was mixed with 2 x SDS sample buffer $(100 \mathrm{mM}$ Tris-HCl, pH 6.8, 10 mM EDTA, 4\% SDS, 10\% glycine) and separated using 10\% SDS-PAGE (Bio-Rad Laboratories, Inc.). Electrophoresed proteins were transferred onto polyvinylidene fluoride membranes (Merck KGaA, Darmstadt, Germany). 
The membranes were blocked with 5\% skimmed milk in PBS for $1 \mathrm{~h}$ at room temperature and were incubated with primary antibodies against iASPP (cat no. ab34898; 1:5,000; Abcam, Cambridge, MA, USA), KLF4 (cat no. ab106629; 1:5,000; Abcam) and $\beta$-actin (cat no. MAB1501; 1:5,000, Merck KGaA) overnight at $4^{\circ} \mathrm{C}$. Membranes were washed three times with Tris-buffered saline with $0.1 \%$ Tween-20 (TBST) for 5 min and incubated with horseradish peroxidase-conjugated goat anti-mouse or anti-rabbit immunoglobulin (cat no. sc2004; 1:5,000; Santa Cruz Biotechnology, Inc., Dallas, TX, USA) for $1 \mathrm{~h}$ at room temperature, and then washed three times for $5 \mathrm{~min}$ with TBST. Protein bands were visualized using an enhanced chemiluminescence reagent (EMD Millipore, Billerica, MA, USA) and quantified by densitometry using Quantity One Image Analysis Software (Version 4.6.7; Bio-Rad Laboratories, Inc.).

MTT assay. Cell proliferation levels were evaluated using an MTT assay. In brief, MKN45 cells were seeded in a 96-well plate at $2 \times 10^{3}$ cells/well for 24,48 and $72 \mathrm{~h}$. MTT solution (20 $\mu \mathrm{l}$ of $5 \mathrm{mg} / \mathrm{ml} \mathrm{MTT}$ ) was added to the cells (for a total culture volume of $200 \mathrm{ml}$ ) prior to incubation at $37^{\circ} \mathrm{C}$ for an additional $4 \mathrm{~h}$. The formazan crystals that formed were dissolved in $100 \mu \mathrm{l}$ dimethylsulfoxide. The absorbance values were read at $560 \mathrm{~nm}$.

Colony formation assay. The colony forming ability of the MKN45 cells was detected using a colony formation assay. The target cells were seeded at a low density (500 cells/plate) in a 6-well plate and cultured until visible colonies appeared ( 2 weeks). The cell colonies were then stained with Giemsa solution for $15 \mathrm{~min}$ at room temperature. The colonies containing $>50$ cells were counted as positive.

Flow cytometric analysis of apoptosis. MKN45 cells were collected and washed twice with ice-cold PBS. The apoptosis rate of the cells was detected with Annexin V-fluorescein and propidium iodide double staining (Apoptosis kit; eBioscience; Thermo Fisher Scientific, Inc.), according to the manufacturer's protocol. Flow cytometric analysis was performed and data were analysed using a FACSCalibur flow cytometer (BD Biosciences, Franklin Lakes, NJ, USA). Flow cytometry analysis was repeated at least 3 times using Windows Multiple Document Interface Flow Cytometry Application (WinMDI version 2.9; Microsoft, Redmond, WA, USA).

Xenograft experiments. A total of 36 athymic nude mice (male, 6- to 8-week-old, 20-30 g in body weight), were obtained from the Animal Experimental Centre of Chongqing Medical University (Chongqing, China). The mice were acclimated for 2 weeks with ad libitum access to standard food and water. They were maintained in an isolated pathogen-free ventilation chamber with a $12 \mathrm{~h}$ light-dark cycle at a temperature of $22 \pm 2^{\circ} \mathrm{C}$ and $40-50 \%$ relative humidity. To establish the gastric cancer model, equal numbers of MKN45 cells $\left(1 \times 10^{6}\right)$ were injected subcutaneously into the right rear flank of each mouse. The mice were divided into four groups (3 mice/group): A negative-control group (injected with control-transfected MKN45 cells), KLF4-overexpression group (injected with KLF4-overexpression-shRNA transfected MKN45 cells),
iASPP-downregulation group (injected with iASPP-inhibition-shRNA transfected MKN45 cells) and combined KLF4/iASPP overexpression group (injected with MKN45 cells overexpressed KLF4 and iASPP). Tumor growth was observed daily in each group and tumor diameter was measured once a week using callipers. Tumor volume was calculated with the following formula: Tumor volume $=\left(\mathrm{Lx} \mathrm{S}^{2}\right) / 2$, where $\mathrm{L}$ is the longest tumor axis and $\mathrm{S}$ is the shortest tumor axis. At 4 weeks post-injection, the mice were sacrificed, or when the maximum tumor diameter reached $2.0 \mathrm{~cm}$ and the tumors were used for further analysis. This study was conducted in accordance with the recommendations of the Guide for the Care and Use of Laboratory Animals of Chongqing Medical University. All animal experiments were approved by the Committee on the Ethics of Animal Experiments of Chongqing Medical University and Chongqing Cancer Hospital. All surgeries were performed under sodium pentobarbital anesthesia and all efforts were made to minimize suffering.

Statistical analysis. Data were analyzed using SPSS software (version 18.0; SPSS, Inc., Chicago, IL, USA). Data are expressed as the mean \pm standard deviation. Results were analyzed using one-way analysis of variance with a least significant difference test for post hoc analysis. $\mathrm{P}<0.05$ was considered to indicate a statistically significant difference.

\section{Results}

Expression of KLF4 and iASPP in GC cells. The expression of KLF4 and iASPP was detected in normal (GES1) and GC cell lines (MKN45, BGC823 and SGC7901) using RT-PCR and western blot analysis. The results demonstrated that the mRNA and protein expression of KLF4 was significantly downregulated in GC cells compared with that in GES1 cells (Fig. 1A and C/E). However, the mRNA and protein expression of iASPP was upregulated in GC cells compared with that in GES1 cells (Fig. 1B and D/F). Additionally, as there was a negative association between the expression of KLF4 and iASPP particularly in MKN45 cells, which is why the MKN45 cell line was selected for subsequent experiments (Fig. 1A-D).

Expression of KLF4 and iASPP in MKN45 cells following lentiviral transfection. In order to overexpress KLF4 in MKN45 cells, cells were transfected with KLF4-overexpression-shRNA lentivirus. Following transfection and selection, expression of KLF4 and iASPP was evaluated in MKN45 cells using western blot analysis. The results demonstrated that the expression of KLF4 was significantly upregulated following KLF4 overexpression via lentiviral delivery (Fig. 2A). Additionally, expression of iASPP was downregulated following overexpression of KLF4 (Fig. 2B). Next, MKN45 cells were transfected with iASPP-inhibition-shRNA lentivirus and expression of iASPP was evaluated using western blot analysis. The results demonstrated that the expression of iASPP was significantly downregulated following lentiviral transfection (Fig. 2C). Next, an iASPP-overexpression-shRNA lentivirus was transfected into the KLF4-overexpressing MKN45 cells. Following transfection, iASPP expression was evaluated using western 
A
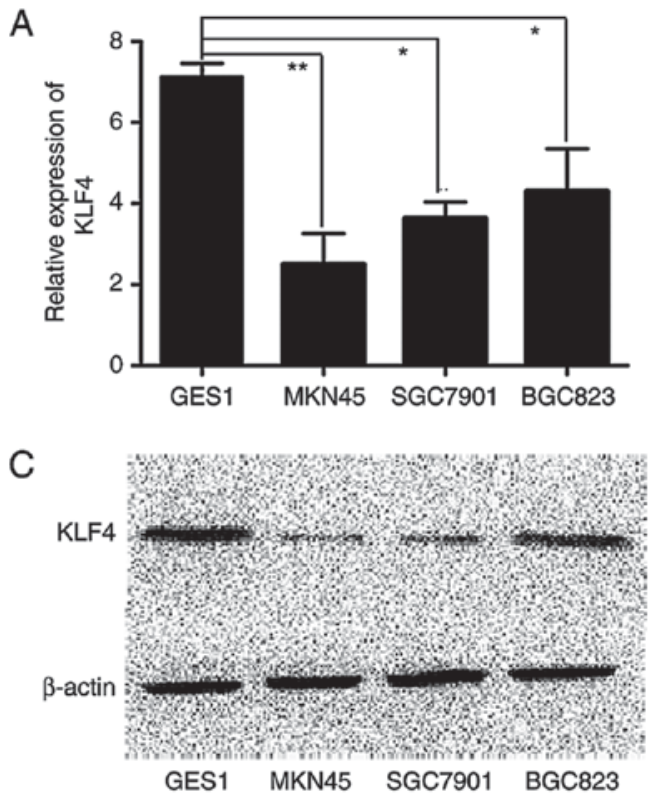

E

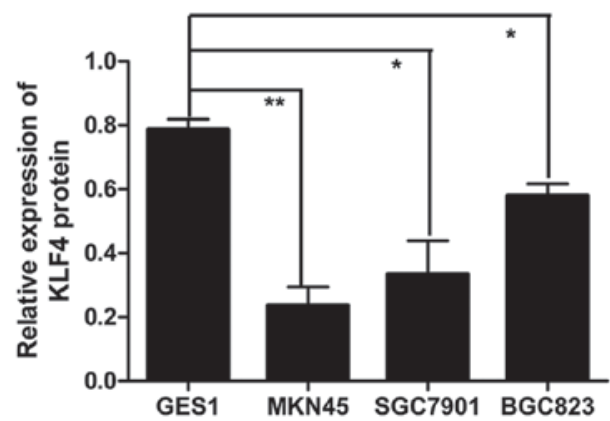

B

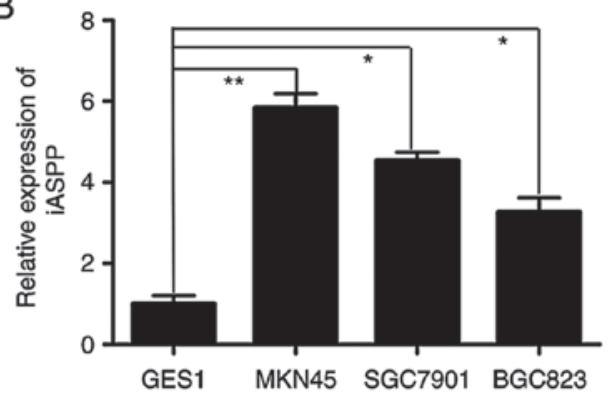

D

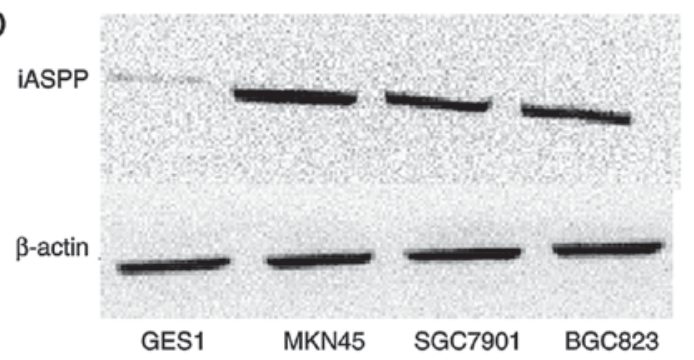

$\mathbf{F}$

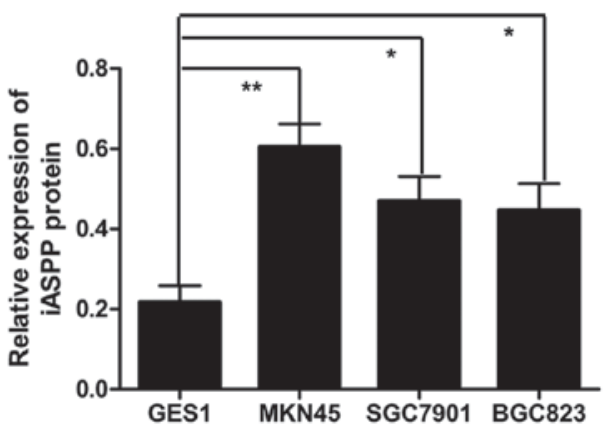

Figure 1. KLF4 is downregulated and iASPP is upregulated in GC cell lines (MKN45, SGC7901 and BGC823). (A) KLF4 mRNA levels were downregulated in the three GC cell lines compared with that in normal GES1 cells. (B) iASPP mRNA levels were upregulated in the three GC cell lines compared with that in normal GES1 cells. (C) Protein expression of KLF4 were downregulated in the three GC cell lines compared with that in normal GES1 cells. (D) The protein expression levels of iASPP were upregulated in the three GC cell lines compared with that in normal GES1 cells. (E) Quantified protein expression levels of KLF4. (F) Quantified protein expression levels of iASPP. ${ }^{* *} \mathrm{P}<0.01$ and ${ }^{*} \mathrm{P}<0.05$ with comparisons shown by lines. GC, gastric cancer; KLF4, kruppel-like factor 4; iASPP, inhibitor of apoptosis-stimulating protein of p53.

A

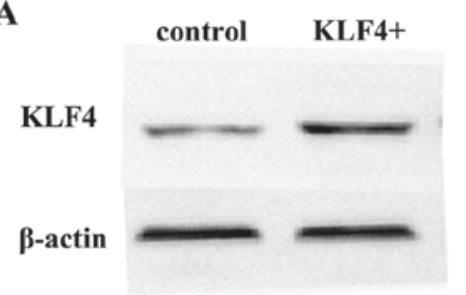

C

iASPP
contro
iASPP-

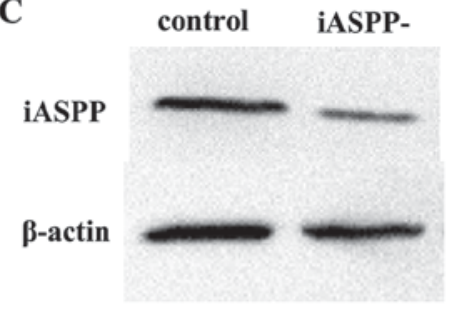

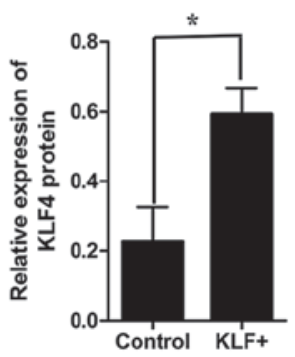

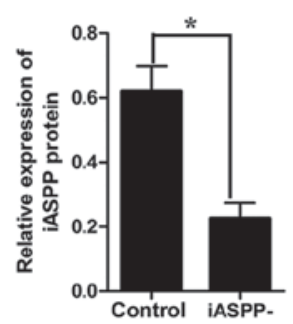

B
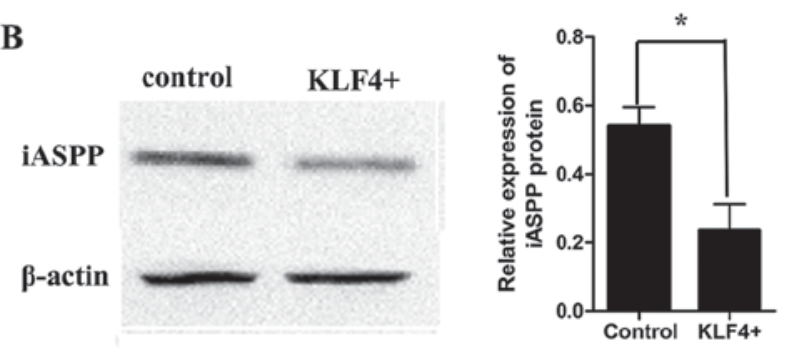

D

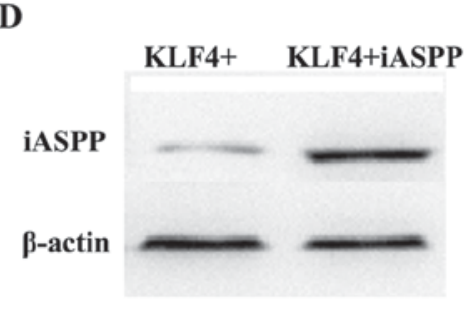

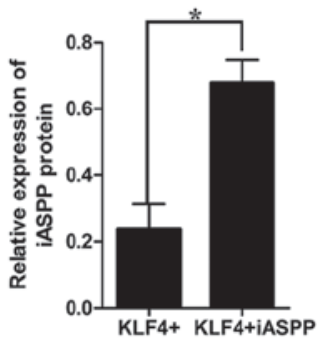

Figure 2. Expression of KLF4 and iASPP following lentiviral transfection. (A) KLF4 expression was upregulated following overexpression of KLF4 by lentiviral delivery. (B) iASPP expression was downregulated following KLF4 overexpression by lentiviral delivery. (C) iASPP expression was upregulated following overexpression of iASPP by lentiviral delivery. (D) iASPP expression was downregulated following downregulation of iASPP by lentiviral delivery. "P<0.05 with comparisons shown by lines. GC, gastric cancer; KLF4, kruppel-like factor 4; iASPP, inhibitor of apoptosis-stimulating protein of p53; +, overexpression; -, downregulation. 

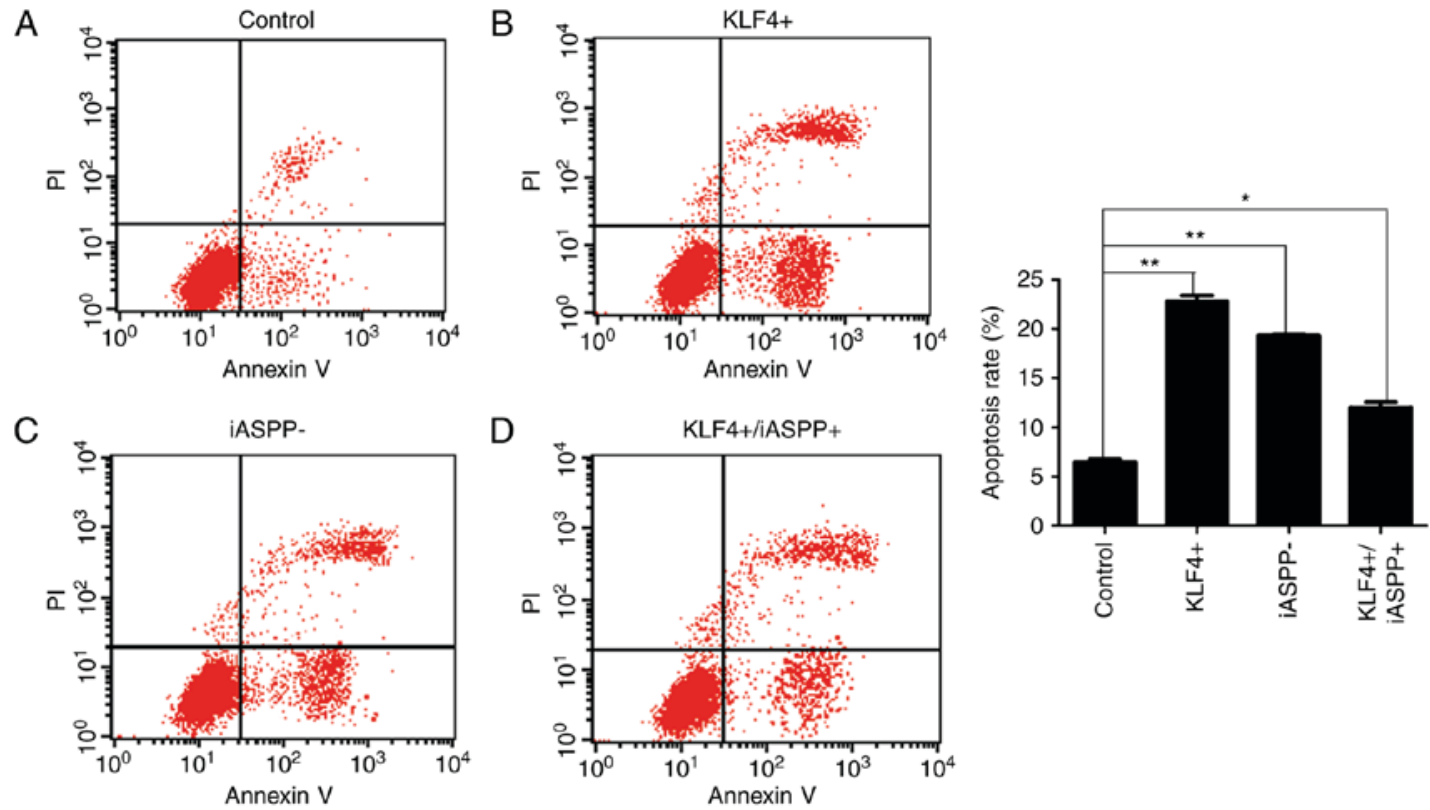

Figure 3. Overexpression of KLF4 and downregulation of iASPP promote apoptosis of GC cells, but the apoptosis level is restored by upregulation of iASPP. (A) The apoptosis level of control GC cells. (B) The apoptosis level of GC cells following upregulation of KLF4. (C) The apoptosis level of GC cells following downregulation of iASPP. (D) The apoptosis level of GC cells following combined upregulation of KLF4 and iASPP. "P $<0.05$ and ${ }^{* *} \mathrm{P}<0.01$ with comparisons shown by lines. GC, gastric cancer; KLF4, kruppel-like factor 4; iASPP, inhibitor of apoptosis-stimulating protein of p53; PI, propidium iodide; +, overexpression; -, downregulation.
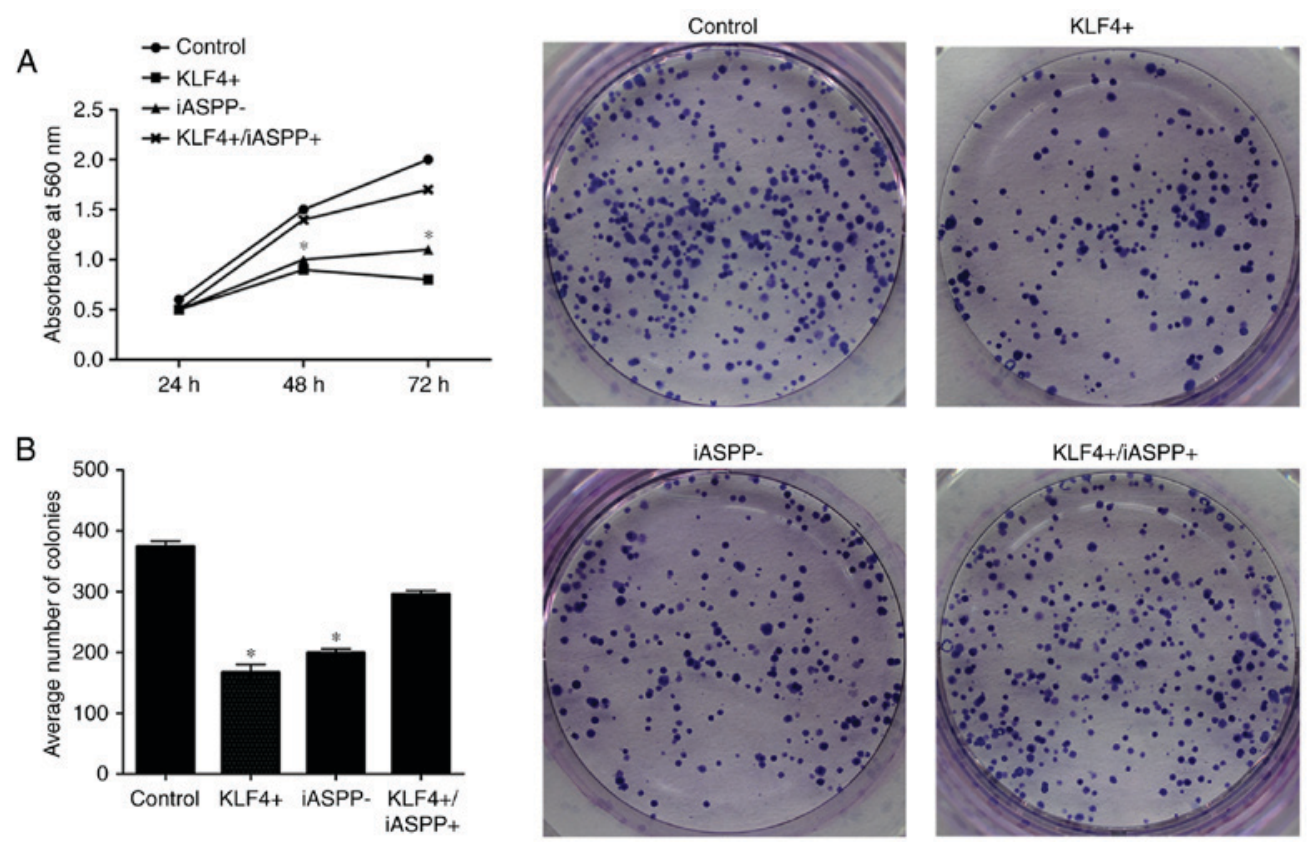

Figure 4. Overexpression of KLF4 and downregulation of iASPP inhibit the proliferation of GC cells, which is restored following upregulation of the expression of iASPP. (A) Proliferation of GC cells was determined using a MTT assay. Proliferation was inhibited in response to upregulation of KLF4 or downregulation of iASPP, but it was restored in response to upregulation of iASPP. (B) The colony-forming ability of GC cells was inhibited by upregulation of KLF4 or downregulation of iASPP, but was restored by upregulation of iASPP. "P $<0.05$ vs. control. GC, gastric cancer; KLF4, kruppel-like factor 4; iASPP, inhibitor of apoptosis-stimulating protein of p53; +, overexpression; -, downregulation.

blot analysis. The results demonstrated that iASPP was upregulated in iASPP-overexpressing MKN45 cells compared with that in the non-transfected cells which overexpressed KLF4 but not iASPP (Fig. 2D).

Apoptosis of MKN45 cells following lentiviral transfection. Flow cytometric analysis of apoptosis was applied in order to determine the function of KLF4 in regulating iASPP in MKN45 cells. The results demonstrated that overexpression of KLF4 and downregulation of iASPP led to an increased rate of apoptosis compared with that in the control MKN45 cells (Fig. 3A-C). The apoptosis rate was partially restored by overexpression of iASPP, which inhibited apoptosis in the MKN45 cells (Fig. 3D). 

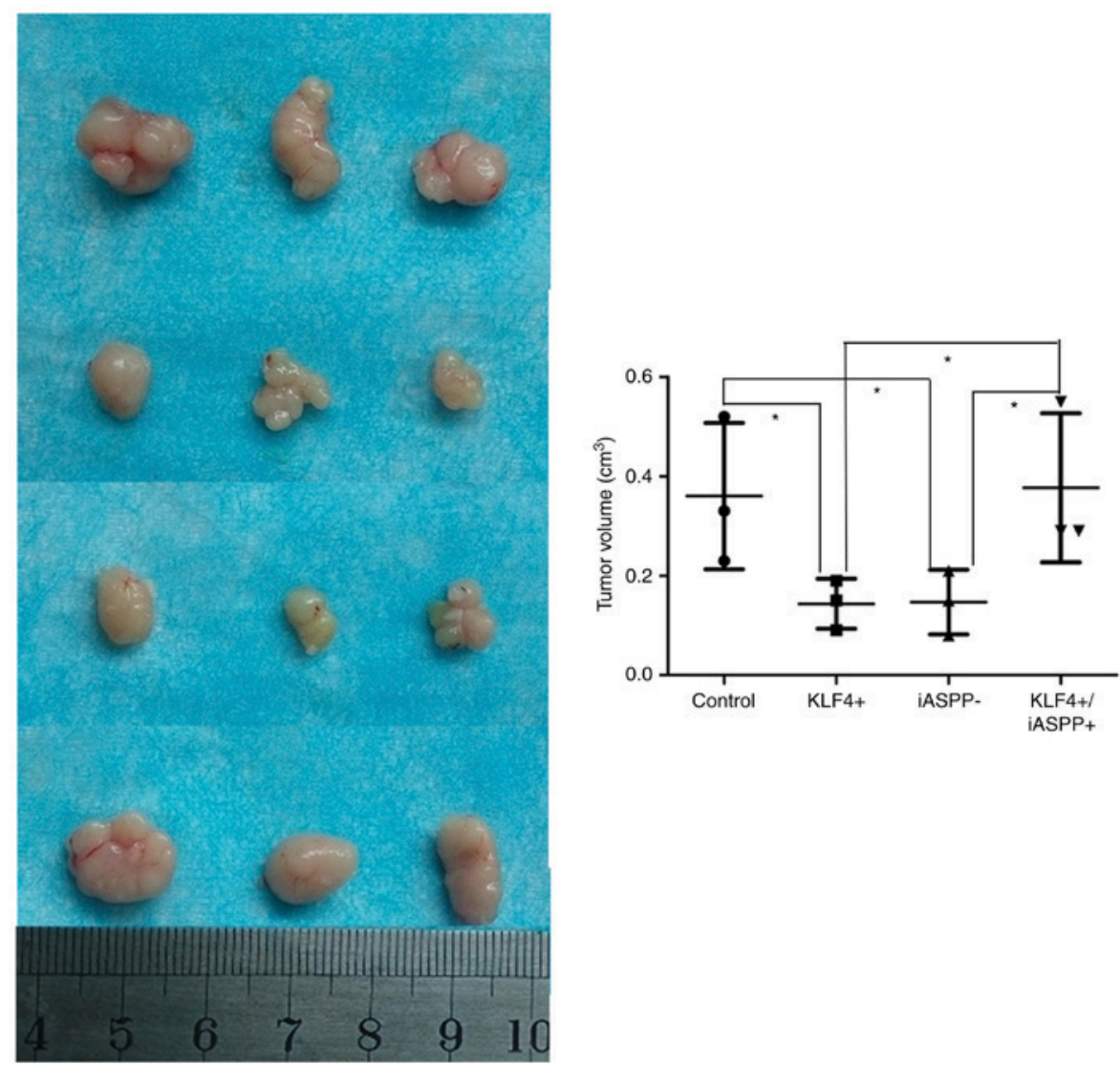

Figure 5. In vivo tumor growth. Growth of GC tumors was significantly inhibited in a xenograft model following upregulation of KLF4 or downregulation of iASPP expression, but the upregulation of iASPP restored the effects of the upregulation of KLF4. "P<0.05. GC, gastric cancer; KLF4, kruppel-like factor 4; iASPP, inhibitor of apoptosis-stimulating protein of p53; +, overexpression; -, downregulation.

Proliferation and colony-forming ability of MKN45 cells following lentiviral transfection. MTT and colony formation assays were employed to evaluate the viability and colony-forming ability of MKN45 cells, respectively. As presented in Fig. 4, overexpression of KLF4 or downregulation of iASPP inhibited the proliferation and colony-forming ability of the MKN45 cells, whereas combined KLF4 and iASPP overexpression significantly promoted the proliferation and colony-forming ability of the cells compared with KLF4 overexpressing alone ( $\mathrm{P}<0.05$; Fig. 4$)$.

In vivo tumor growth following lentiviral transfection. To investigate the function of KLF4 and iASPP on tumor growth in vivo, equal numbers of MKN45 cells $\left(10^{6}\right.$ cells/mouse) were subcutaneously injected into nude mice. Tumors were evident in all mice. As presented in Fig. 5, overexpression of KLF4 and downregulation of iASPP significantly inhibited tumor growth in vivo, whereas combined KLF4 and iASPP overexpression promoted the growth of tumors in vivo (Fig. 5).

\section{Discussion}

Wild-type p53 may act as a tumor suppressor gene and inhibit the malignant transformation of cells, whereas the mutant-type p53 may significantly contribute to the development of cancer. A previous study confirmed that mutations in the p53 gene are not a predominant event in gastric carcinogenesis $(12,13)$. The molecular mechanism behind the occurrence of gastric cancer expressing wild-type p53 is therefore important. In the present study, the expression levels of KLF4 and iASPP were evaluated in GC cell lines with wild-or mutant-type of p53 (MKN45, BGC823 and SGC7901 cells) and in normal gastric mucosa GES1 cells. It was demonstrated that expression of KLF4 was significantly decreased and expression of iASPP was increased in GC cells compared with that in GES1 cells, but particularly in the wild-type p53-expressing cell line MKN45. Previous studies have demonstrated that KLF4 acts as a tumor suppressor and inhibits the proliferation of GC cells $(17,22)$. The results of the present study demonstrated that proliferation of the MKN45 cells was significantly inhibited, whereas apoptosis was promoted following overexpression of KLF4, which is consistent with previous findings.

iASPP is an evolutionarily conserved inhibitor of wild-type p53 (4) and overexpression of iASPP has been observed in several types of human cancer (8-11). The results of the present study demonstrated that the expression levels of iASPP were increased in GC cells compared with those in GES1 cells. These results suggest that iASPP and KLF4 may serve as independent prognostic markers for tumor cell proliferation in GC. Previous studies have confirmed that the knockdown of iASPP may significantly inhibit tumor growth and cell proliferation in various types of cancer, including glioblastoma (35), prostate cancer (36) and lung cancer (37). The present study demonstrated that changes in the expression of iASPP were associated with proliferation and apoptosis rates of MKN45 cells. The imbalance between proliferation and apoptosis contributes to 
the formation and development of human tumors (38). The downregulation of iASPP decreased the proliferation and colony-forming ability of the MKN45 cells. Additionally, downregulation of iASPP promoted cell apoptosis. These results suggest that deregulated expression of iASPP may contribute to tumorigenesis in GC cells that express wild-type p53. These results are consistent with previous studies on leukaemia and non-small cell lung cancer in which iASPP is highly expressed $(39,40)$.

The iASPP-mediated signaling pathway that regulates cancer cell proliferation remains unclear. The present study demonstrated that the expression of KLF4 was significantly decreased and that the expression of iASPP was significantly increased in GC cells, particularly MKN45 cells. It has been indicated that KLF4 and p53 may interact with each other, whereas iASPP is an evolutionarily conserved inhibitor of p53 $(7,28,29)$. Since there may be a functional association between KLF4, iASPP and p53, the aim of the present study was to investigate the association between KLF4 and iASPP in GC. In the present study, the expression of iASPP was evaluated in response to the overexpression of KLF4 (achieved using lentiviral delivery). The results demonstrated that overexpression of KLF4 led to downregulation of iASPP, suggesting that KLF4 may regulate the expression of iASPP. Additionally, overexpression of KLF4 inhibited the proliferation and colony formation ability of the MKN45 cells. Since the decrease in KLF4 expression led to increased expression iASPP in GC, the present study investigated whether low levels of KLF4 and increased levels of iASPP may promote cellular proliferation in GC. Next, iASPP was overexpressed following KLF4 expression by using specific shRNAs in order to further validate the association between KLF4 and iASPP. The results demonstrated that overexpression of iASPP inhibited the effects of KLF4 in MKN45 cells. Increased expression levels of KLF4 inhibited the proliferation and promoted the apoptosis of MKN45 cells, whereas KLF4-mediated effects were reversed by overexpression of iASPP. These results suggest that KLF4 may act as a potential tumor suppressor by inhibiting the expression of iASPP in GC. Additionally, tumor growth was significantly inhibited in response to the downregulation of iASPP or upregulation KLF4 in vivo. However, in vivo KLF4-mediated effects were reversed by iASPP overexpression. These results suggest that this decrease in tumor growth may be due to the decrease in the expression of iASPP, which was caused by KLF4 overexpression. Previous studies have indicated a novel molecular mechanism of action for KLF4 and iASPP in GC (41). However, in the present study, the association between KLF4, iASPP and p53 was not further investigated. Therefore, future studies are required to investigate the function of this signaling pathway in the development of GC, employing GC cells that express the wild-type p53. Additionally, since the ASPP family contains three members (iASPP, ASPP1 and ASPP2), which share similar sequences in their C-terminus domain (42), there may be also an association between KLF4 and ASPP1 or ASPP2.

KLF4 may act as a tumor suppressor that may inhibit cellular proliferation, whereas iASPP may act as an oncogene that promotes the proliferation of GC cells that express the wild-type p53. In the present study, it was demonstrated that KLF4 inhibited cell proliferation and colony formation ability partly by targeting iASPP. Therefore, the KLF4/iASPP axis may serve a vital function in regulating the proliferation of $\mathrm{GC}$ cell, and provide a potential diagnostic and therapeutic target for $\mathrm{GC}$.

\section{Acknowledgements}

The authors would like to thank Professor Dong-Lin Wang (Chongqing Cancer Hospital, Chongqing, China), for providing mentor support, incisive comments and useful suggestions.

\section{Funding}

The present study was supported by the special fund for Postdoctoral Scientific Research Projects (grant no. Xm2017091).

\section{Availability of data and materials}

The datasets generated and analyzed in the present study are included in this published article.

\section{Authors' contributions}

LLW contributed to the study design, experimental work, data analysis and preparation of the manuscript. DLW, YL, LCL, ZJW and HWM participated in the design of the study and experimental work. YZW, HQY and DY performed the statistical analysis. DLW participated in the study design and coordination, and contributed to the drafting of the manuscript. All authors read and approved the final manuscript.

\section{Ethics and consent to participate}

The study was approved by the Ethics Committee of Chongqing Cancer Hospital and Chongqing Medical University, and all patients provided informed consent for participation in the present study.

\section{Consent for publication}

The study participants provided consent for publication.

\section{Competing interests}

The authors declare that they have no competing interests.

\section{References}

1. Siegel RL, Miller KD and Jemal A: Cancer statistics, 2016. CA Cancer J Clin 66: 7-30, 2016.

2. Carcas LP: Gastric cancer review. J Carcinog 13: 14, 2014.

3. Chen J, Xie F, Zhang L and Jiang WG: iASPP is over-expressed in human non-small cell lung cancer and regulates the proliferation of lung cancer cells through a p53 associated pathway. BMC Cancer 10: 694, 2010.

4. Dong P, Ihira K, Hamada J, Watari H, Yamada T, Hosaka M, Hanley SJ, Kudo M and Sakuragi N: Reactivating p53 functions by suppressing its novel inhibitor iASPP: A potential therapeutic opportunity in p53 wild-type tumors. Oncotarget 6: 19968-19975, 2015. 
5. Duffy MJ, Synnott NC, McGowan PM, Crown J, O'Connor D and Gallagher WM: p53 as a target for the treatment of cancer. Cancer Treat Rev 40: 1153-1160, 2014.

6. Meek DW: Regulation of the p53 response and its relationship to cancer. Biochem J 469: 325-346, 2015.

7. Laska MJ, Vogel UB, Jensen UB and Nexø BA: p53 and PPP1R13L (alias iASPP or RAI) form a feedback loop to regulate genotoxic stress responses. Biochim Biophys Acta 1800: 1231: 1240, 2010.

8. Wang C, Gao C, Chen Y, Yin J, Wang P and Lv X: Expression pattern of the apoptosis-stimulating protein of p53 family in $\mathrm{p}^{+} 3^{+}$human breast cancer cell lines. Cancer Cell Int 13: 116, 2013.

9. Liu H, Wang M, Diao S, Rao Q, Zhang X, Xing H and Wang J: siRNA-mediated down-regulation of iASPP promotes apoptosis induced by etoposide and daunorubicin in leukemia cells expressing wild-type p53. Leuk Res 33: 1243-1248, 2009.

10. Chen J, Xie F, Zhang L and Jiang WG: iASPP is over-expressed in human non-small cell lung cancer and regulates the proliferation of lung cancer cells through a p53 associated pathway. BMC Cancer 10: 694, 2010.

11. Lin BL, Xie DY, Xie SB, Xie JQ, Zhang XH, Zhang YF and Gao ZL: Down-regulation of iASPP in human hepatocellular carcinoma cells inhibits cell proliferation and tumor growth. Neoplasma 58: 205-210, 2011.

12. Meng WD, Chu RX, Wang BZ, Wang LP, Ma LL and Wang LX: Helicobacter pylori infection and expressions of apoptosis-related proteins p53, ASPP2 and iASPP in gastric cancer and precancerous lesions. Pathol Biol 61: 199-202, 2013.

13. Berloco P, Russo F, Cariola F, Gentile M, Giorgio P, Caruso ML, Valentini AM, Di Matteo G and Di Leo A: Low presence of $p 53$ abnormalities in $\mathrm{H}$. pylori-infected gastric mucosa and in gastric adenocarcinoma. J Gastroenterol 38: 28-36, 2003.

14. Cai Y, Qiu S, Gao X, Gu SZ and Liu ZJ: iASPP inhibits p53-independent apoptosis by inhibiting transcriptional activity of $\mathrm{p} 63 / \mathrm{p} 73$ on promoters of proapoptotic genes. Apoptosis 17 : 777-783, 2012.

15. Gillotin S and Lu X: The ASPP proteins complex and cooperate with p300 to modulate the transcriptional activity of p53. FEBS Lett 585: 1778-1782, 2011.

16. Lee HY, Ahn JB, Rha SY, Chung HC, Park KH, Kim TS, Kim NK and Shin SJ: High KLF4 level in normal tissue predicts poor survival in colorectal cancer patients. World J Surg Oncol 12: 232, 2014.

17. Ghaleb AM and Yang VW: Krüppel-like factor 4 (KLF4): What we currently know. Gene 611: 27-37, 2017.

18. Foster KW, Ren S, Louro ID, Lobo-Ruppert SM, McKie-Bell P, Grizzle W, Hayes MR, Broker TR, Chow LT and Ruppert JM: Oncogene expression cloning by retroviral transduction of adenovirus E1A-immortalized rat kidney RK3E cells: Transformation of a host with epithelial features by c-MYC and the zinc finger protein GKLF. Cell Growth Differ 10: 423-434, 1999.

19. Foster KW, Frost AR, McKie-Bell P, Lin CY, Engler JA, Grizzle WE and Ruppert JM: Increase of GKLF messenger RNA and protein expression during progression of breast cancer. Cancer Res 60: 6488-6495, 2000.

20. Ton-That H, Kaestner KH, Shields JM, Mahatanankoon CS and Yang VW: Expression of the gut-enriched Krüppel-like factor gene during development and intestinal tumorigenesis. FEBS Lett 419: 239-243, 1997.

21. Zhao W, Hisamuddin IM, Nandan MO, Babbin BA, Lamb NE and Yang VW: Identification of Krüppel-like factor 4 as a potential tumor suppressor gene in colorectal cancer. Oncogene 23: 395-402, 2004

22. Hsu LS, Chan CP, Chen CJ, Lin SH, Lai MT, Hsu JD, Yeh KT and Soon MS: Decreased s-like factor 4 (KLF4) expression may correlate with poor survival in gastric adenocarcinoma. Med Oncol 30: 632, 2013.

23. Ohnishi S, Ohnami S, Laub F, Aoki K, Suzuki K, Kanai Y, Haga K, Asaka M, Ramirez F and Yoshida T: Downregulation and growth inhibitory effect of epithelial-type Krüppel-like transcription factor KLF4, but not KLF5, in bladder cancer. Biochem Biophys Res Commun 308: 251-256, 2003.
24. Yang WT and Zheng PS: Kruppel-like factor 4 function as a tumor suppressor in cervical carcinoma. Cancer 118: 3691-3702, 2012.

25. Krstic M, Stojnev S, Jovanovic L and Marjanovic G: KLF4 expression and apoptosis-related markers in gastric cancer. J BUON 18: 695-702, 2013.

26. Kaczynski J, Cook T and Urrutia R: Sp1- and Krüppel-like transcription factors. Genome Biol 4: 206, 2003.

27. Black AR, Black JD and Azizkhan-Clifford J: Sp1 and krüppel-like factor family of transcription factors in cell growth regulation and cancer. J Cell Physiol 188: 143-160, 2001.

28. Elemento O: KLF4: A new player in plasma cell development. Cell Cycle 15: 2691-2692, 2016.

29. Brosh R, Assia-Alroy Y, Molchadsky A, Bornstein C, Dekel E, Madar S, Shetzer Y, Rivlin N, Goldfinger N, Sarig R and Rotter V: p53 counteracts reprogramming by inhibiting mesenchymal-to-epithelial transition. Cell Death Differ 20: 312-320, 2013.

30. Yokozaki H: Molecular characteristics of eight gastric cancer cell lines established in Japan. Pathol Int 50: 767-777, 2000.

31. Sigal A and Rotter V: Oncogenic mutations of the p53 tumor suppressor: The demons of the guardian of the genome. Cancer Res 60: 6788-6793, 2000

32. Zhang SW, Xiao SW and Lü YY: Adenovirus-mediated P53 cancer transfer increases the thermosensitivity of human gastric carcinoma cell lines in vitro and in vivo. Chin J Cancer Res 15 : 107-111, 2003.

33. Liu J, Zhang Y, Xie YS, Wang FL, Zhang LJ, Deng T and Luo HS: 5-Aza-2'-deoxycytidine induces cytotoxicity in BGC-823 cells via DNA methyltransferase 1 and $3 \mathrm{a}$ independent of p53 status. Oncol Rep 28: 545-552, 2012.

34. Livak KJ and Schmittgen TD: Analysis of relative gene expression data using real-time quantitative PCR and the $2^{-\Delta \Delta C \mathrm{~T}}$ method. Methods 25: 402-408, 2001.

35. Li G, Wang R, Gao J, Deng K, Wei J and Wei Y: RNA interference-mediated silencing of iASPP induces cell proliferation inhibition and G0/G1 cell cycle arrest in U251 human glioblastoma cells. Mol Cell Biochem 350: 193-200, 2011.

36. Chen J, Xiao H, Huang Z, Hu Z, Qi T, Zhang B and Liu SH: MicroRNA124 regulate cell growth of prostate cancer cells by targeting iASPP. Int J Clin Exp Pathol 7: 2283-2290, 2014.

37. Li S, Shi G, Yuan H, Zhou T, Zhang Q, Zhu H and Wang X: Abnormal expression pattern of the ASPP family of proteins in human non-small cell lung cancer and regulatory functions on apoptosis through p53 by iASPP. Oncol Rep 28: 133-140, 2012.

38. Grabenbauer GG, Suckorada O, Niedobitek G, Rödel F, Iro H, Sauer R, Rödel C, Schultze-Mosgau S and Distel L: Imbalance between proliferation and apoptosis may be responsible for treatment failure after postoperative radiotherapy in squamous cell carcinoma of the oropharynx. Oral Oncol 39: 459-469, 2003.

39. Liu H, Wang M, Diao S, Rao Q, Zhang X, Xing H and Wang J: siRNA-mediated down-regulation of iASPP promotes apoptosis induced by etoposide and daunorubicin in leukemia cells expressing wild-type p53. Leuk Res 33: 1243-1248, 2009.

40. Chen J, Xie F, Zhang L and Jiang WG: iASPP is over-expressed in human non-small cell lung cancer and regulates the proliferation of lung cancer cells through a p53 associated pathway. BMC Cancer 10: 694, 2010.

41. Wang LL, Xu Z, Peng Y, Li LC and Wu XL: Downregulation of inhibitor of apoptosis-stimulating protein of p53 inhibits proliferation and promotes apoptosis of gastric cancer cells. Mol Med Rep 12: 1653-1658, 2015.

42. Sullivan A and Lu X: ASPP: A new family of oncogenes and tumour suppressor genes. Br J Cancer 96: 196-200, 2007.

This work is licensed under a Creative Commons Attribution-NonCommercial-NoDerivatives 4.0 International (CC BY-NC-ND 4.0) License. 